

$$
\text { L }
$$




\title{
Cepstral Filtering on a Columnar \\ Image Architecture: A Fast \\ Algorithm for Binocular Stereo \\ Segmentation.
}

by

Yehezkel Yeshurun $\dagger$

Eric L. Schwartz $\ddagger \dagger$

Technical Report No. 286

Robotics Report No. 106

March, 1987

\author{
$\ddagger$ New York University \\ Dept. of Computer Science \\ Courant Institute of Mathematical Sciences \\ 251 Mercer Street \\ New York, New York 10012 \\ † Computational Neuroscience Laboratories \\ Dept. of Psychiatry \\ NYU Medical Center \\ 550 First Ave \\ New York, N.Y. 10016
}

Work on this paper has been supported by the System Development Foundation, AFOSR \#85-0341, and the Nathan S. Kline Research Institute. 



\begin{abstract}
Many primate visual cortex architectures (including the human) have a prominent feature responsible for the mixing of left and right eye visual data: ocular dominance columns represent thin ( about 6 minutes of arc) strips of alternating left and right eye input to the brain. In the present paper we show that such an architecture, when operated upon with a cepstral filter, provides a strong cue for binocular stereopsis. Specifically, the vector of binocular disparity may be easily identified in the output of the (columnar based) cepstral filter. This algorithm is illustrated with application to a random dot stereogram and to natural images. We suggest that this provides a fast algorithm for stereo segmentation, in a machine vision context. In a biological context, this may provide a computational rationale for the existence of columnar systems, both with regard to ocular mixing, and to other visual modalities which have a columnar architecture.
\end{abstract}





\section{Introduction}

In this paper, an algorithm is presented which provides a fast, one step analysis of the binocular disparity of a pair of stereo images. This work is strongly motivated by architectural features of the visual cortex of monkeys and humans, and it has a close relationship to certain limitations and advantages which are shared with human stereo vision. Free use has been made of anatomical and psychophysical data, in the explanation and discussion of the algorithm. There is no attempt in the present work to construct a model of human stereopsis. On the contrary, we propose this work as a favorable algorithm for computational stereo applications.

Among the many cues which humans use for inferring the structure of a three dimensional scene, binocular stereopsis has been one of the most intensively investigated (both algorithmically and psychophysically). This cue is based on an (unknown) means of utilization of the slightly different views of a three dimensional scene, as projected onto the right and left retina. From a generic point of view, this problem reduces to a matching or correlation of two slightly different scenes, in order to find the (vector) displacement of small corresponding patches of projected image.

Although apparently simple, fast solutions to this problem have been elusive. Many proposed algorithms are based on a direct search for matching features in the left and right half-images. Some recent approaches base this search on a relaxation, or variational approach, possibly utilizing multi-scale image data structures (e.g.[1]). However, it has been claimed[2] that inherently sequential approaches to early vision, such as relaxation (or cooperative) algorithms, are biologically implausible. The low pulse rate of cortical neurons, together with the very rapid response time of biological stereo segmentation argue against a sequential approach to stereo segmentation. Also, from a machine vision viewpoint, it would seem desirable to have a "one-shot" fast algorithm for stereo segmentation.

The present algorithm achieves this goal by using local context, in parallel, to arrive at a "one shot" measurement of the disparity vector. A windowed cepstral filter, operating on an interlaced image format which is inspired by the structure of ocular dominance columns in primate visual cortex, provides this "one-shot" performance. 


\section{CEPSTRAL FILTERING}

The basic idea of the algorithm is to apply a cepstral filter[3] to a stereo image which is formated in a way suggested by the ocular dominance column pattern of primate visual cortex[4]. Specifically, this pattern presents the left and right images of a stereo pair in the form of thin "strips" of image, alternating between left and right half-images. Figure 1 shows an example of a computer graphic reproduction of this pattern, reconstructed in our laboratory from the brain of a macaque monkey.

Cepstral filtering is a well known method of measuring auditory "echo": the power spectrum of the log of the power spectrum of an audio signal with an echo present has a strong and easily identified component which is a direct measure of the echo period[5,3]. The binocular disparity measurement then reduces to that of application of a non-linear local filter (cepstral filter), followed by peak detection. This filter is applied within windows which span an ocular dominance column pair. This approach yields a sirong stereo signal when presented with both natural images and random dot stereograms, and is resistant $w$ inıage degradations such as blur, size difference, and intensity changes.

Because this algorithm is applied within fixed "windows", it cannot resolve changes in binocular disparity which occur within the window size. However, this is in agreement with the properties of primate stereo vision. Tyler has shown that humans cannot resolve changes in binocular disparity which vary over a scale finer than about 10 minutes of $\operatorname{arc}^{1}[6,7]$. This is consistent with our algorithm, as the window size determined by the scale of the ocular dominance column pattern of monkey visual cortex lies within this region of angular size. Thus, the windowing method of our algorithm is consistent with the known spatial resolution of primate stereo vision. Our algorithm, like the human visual system, has extremely high stereo-acuity, but relatively low spatial acuity.

The present algorithm presents several unique properties:

1.) It provides a computational justification for the existence of columnar interlacing, which is a common architectural feature of primate cortex.

2.) It provides a "one shot", or purely parallel algorithm. There is no iterative component. Indeed, this algorithm could be implemented in a straightforward way by means of optical systems. It thus provides

\footnotetext{
${ }^{1}$ Il is necessary here to dislinguish between stereo-acuily, which represents the ability of humans to discriminate two nearby depth planes, and stereo posilional accuracy. Stereo acuity is extremely high (aboul 2 arc seconds): humans can discriminate two stimuli which differ in depth by aboul 100 microns at three foo viewing distance. Slereo positional accuily, however, represents the ability of humans to perceive rapid changes in depth. This cannol be done when the rate of depth change is greater than 1 cycle/10 minutes of arc, which is aboul 10 times coarser than monocular spatial acuily.
} 
a candidate for "real time" performance in stereo segmentation.

3.) It is in agreement with known psychophysical limitations and anatomical properties of stereo vision.

We will now briefly describe some architectural features of the primate visual system, and then the details of the algorithm.

\section{Ocular dominance column pattern}

Many primates, including humans, possess "ocular dominance columns" in primary visual cortex. $[4,8]$. The left and the right eye views of a scene interact for the first time at the level of striate cortex, and this interaction begins with the formating of binocular data as thin ( $0.5 \mathrm{~mm}$ monkey; $1 \mathrm{~mm}$ human) strips of cortex which receive terminals from either the left or right eye. Figure 1 shows a reconstruction of this pattern. Cortical magnification factor in the macaque monkey is estimated to be about $10-20 \mathrm{~mm} / \operatorname{deg}[9,10]$. Since the width of a pair of columns is about $1 \mathrm{~mm}$ in macaque monkeys, the angular extent of a column pair is about 1/10 - 1/20 degree, or 3- 6 minutes of arc. Since visual acuity is about 1 minute of arc, a column pair extends over about 3-6 "resolution" units.

The function, if any, of the ocular dominance column pattern is currently unknown. One of the principle motivations of the present research has been to investigate algorithms which might be related to architectural features of striate cortex, such as this columnar pattern. For purposes of this discussion, the most notable feature of this pattern is that small patches of the left and right eye view of the scene are placed next to one another in layer IV of striate cortex. Figure 2A schematically illustrates this situation. The task of stereo segmentation is to determine the vector displacement of these two image patches.

\section{Cepstral filtering}

There are two aspects to the present algorithm. The first of these depends on the concept of "cepstral filter". The second depends on the use of a columnar image architecture. Appendix A provides a more detailed analysis of some of the complexities which are associated with the "columnar architecture". 


\section{CEPSTRAL FILTERING}

Consider an interlaced image $f(x, y)$ to be composed of a single columnar pair. Also, assume, for simplicity, that there is no binocular disparity, and that the data consist simply of an image patch $s(x, y)$ (the "right-eye" patch), and an identical patch "butted" against it ( the left eye patch). Since there is assumed to be no disparity, the left and right eye images are identical, and the width of a single column is " $D$ ". We can mathematically represent this image pair as follows. (The * operator represents two dimensional convolution.)

$$
f(x, y)=s(x, y) *\{\delta(x, y)+\delta(x-D, y)\}
$$

The power spectrum of such an image pair is

$$
F(u, v)=S(u, v) \cdot\left\{1+e^{-i \pi(D \cdot u)}\right\} .
$$

By forming the logarithm of $F(u, v)$, the product structure becomes a sum:

$$
\log F(u, v)=\log S(u, v)+\log \left(1+e^{-i \pi D \cdot u}\right)
$$

and the spectrum of (3) will have a prominent term located at the magnitude of the shift $(D, 0)$. In Appendix A, we derive the power spectrum of the term $\log \left(1+e^{-i \pi D \cdot u}\right)$, and show that it consists of a principal term at the location $(D, 0)$, and a series of harmonics at integral multiples, with much smaller amplitude.

Equations (1-3) describe a simple case of two identical images, placed side by side ( simulating a small section of "columnar " image). It is easy to extend this situation to cases where a left or right shift of one the image pairs has occurred, in order to simulate "binocular disparity". The same kind of analysis will apply, and it is possible to locate a strong peak in the cepstrum of the image patch whose location is equal to the basic columnar shift $\mathrm{D}$, with an additional term in the disparity added to ( or subtracted from) this shift. This situation is best illustrated with a simple image example.

Figure $2 \mathrm{~A}$ shows a small patch of image. There is a horizontal and vertical component of disparity. Figure $2 \mathrm{~B}$ shows the power spectrum of this image. Figure $2 \mathrm{C}$ shows the cepstrum ( power spectrum of $\log$ power spectrum). In these power spectra, zero frequency has been shifted to the center of the figure, so that there is bilateral symmetry about the origin. There are two bright dots, representing 


\section{Yeshurun Schwartz}

the disparity term in the cepstrum. These dots are made apparent by thresholding the cepstrum, as in figure $2 \mathrm{D}$. Note that the units of the cepstrum are the same as the original physical units of the image. This can be demonstrated by measuring the linear spacing of two features in the image plane (e.g. the eyes), and then measuring the distance from the origin to the peak in the cepstrum. Thus, spatial position in the cepstral is a direct measure of the "disparity" of the left and right half images.

The image dependent terms in the cepstrum (i.e. the cepstrum of the half-images themselves) can be removed easily, since we have access to the two half-images. Figure $2 \mathrm{E}$ shows the cepstrum of the left half image of figure $2 \mathrm{~A}$. Subtracting this cepstrum from the total cepstrum, in figure $2 \mathrm{~F}$, we isolate the disparity vector, shown as two dots whose vector displacement from the origin is the disparity vector.

In practice, we have not found it necessary to subtract the image dependent terms, as shown in figure $2 \mathrm{~F}$. A simple peak detection algorithm has been capable of isolating and measuring the binocular disparity directly from the cepstrum of the columnar image pair, as shown in figure $2 \mathrm{C}$.

The key idea in this work is that the columnar interlacing shifts the disparity term, in the cepstral plane, by an amount " $D$ ", where " $D$ " is the column size. Then, as long as peak detection is restricted to a region of the cepstral plane within $\left[\frac{D}{2}, \frac{3 D}{2}\right]$, it is quite easy to locate this term as a "bright dot" or peak, with no competing areas of high intensity (see Appendix A for a detailed discussion). Thus, in this case, it is a simple matter to measure the disparity. It is quite interesting to note that the present algorithm has its simplest case for disparities within the region of the width of a column, because human vision also finds its easiest stereo task when presented with stereo pairs whose disparity is within a psychophysically defined region called "Panum's area"[11,6]. In humans, Panum's area corresponds to 6 minutes of arc, and it also corresponds to the estimated extent of human ocular dominance columns[12]. These issues are discussed more extensively in the appendix.

\section{Summary of the algorithm}

Given a stereo pair, i.e two images of the same scene, the algorithm is applied as follows:

1. The two images are interlaced to yield pairs of corresponding "patches". These patches can be of 


\section{CEPSTRAL FILTERING}

arbitrary shape and might even overlap, though only non-overlapping rectangular areas are demonstrated here. One might also apply smoothing operators (i.e Hanning windows) to the windowed images, although we have not found it necessary to so, as the stereo signal is extremely strong.

The size of the "window" is essentially determined by the size of the "ocular dominance columns" which cause the interlacing. Based on the human and monkey visual systems, this window size is estimated to be within the range of 6 minutes of arc.

2. Each window is processed (in parallel) by the cepstral operator.

3. The disparity vector is then found by a peak detection algorithm applied to the windowed cepstrum of the stereo pair. For disparities whose magnitude is $<D$, i.e. "Panum's area", the search is restricted to the region $\left[\frac{D}{2}, \frac{3 D}{2}\right]$ in the cepstral plane.

\section{Performance of the algorithm}

The windowed cepstral filter is easy to implement ( it depends on little more than access to an FFT algorithm). Its complexity is $O(N \cdot \log N)$, from the FFT stage. Full parallelism is easy to achieve, since the windowed filter can be run simultaneously ( there is no interaction between neighboring image patches). Since this algorithm relies mainly on an ability to estimate power spectral densities, as well as a simple (logarithmic) non-linearity, it would seem to be easy to implement in the context of optical computation.

Figure 3 shows an example of a natural scene, in which a window of about 6 minutes of arc is indicated. In order to obtain sufficient resolution from this scene, we have photographically expanded this small segment of it, and performed the cepstral analysis on it. This corresponds to a pair of (foveal) ocular dominance column patches. Figure 3C shows the cepstrum of the "columnar" pair of figure $3 \mathrm{~B}$. The cepstral signal is evident as the two bright dots on the $\mathrm{x}$-axis.

It is technically difficult to analyze fully a natural scene at this resolution, since we are essentially working on a scale in which human visual acuity is equivalent to the pixel size of the image. In other work, we show that this requires a (conventional, constant resolution) image of size $16,000 \times$ 16,000 pixels ( see figure 6). A more sensible path at this point would be to use (as does the human 
system) space variant image representations. However, we show in figure 4 a $3500 \times 3500$ random dot stereogram, fully segmented by the present algorithm. This stereo pair depicts a "pac man" that is detectable only by binocular cues. We have scaled this stereogram to match the following parameters of human vision. The size of each box of the stereogram subtends about 8 degrees of field, so that the percept within the box subtends about 5 degree. The size of the windows in the box is 5 minutes of arc. Thus, the positional accuracy of this segmentation is no better than 5 minutes of arc. This lack of fine detail is evident in slight aliasing of the boundaries of the stereo percept. Based on Tyler's measurements, this aliasing, or an equivalent lack of positional detail, should be present in human segmentation of the stereo pair shown in figure $4 .^{2}$

\section{Pre-processing of stereo images}

We emphasize that the input to this algorithm is not necessarily a "gray scale" image, as we have used in the figures. Naturally, any pre-processing ( e.g. high pass filtering, edge enhancement, etc.) is compatible with this approach. However, it is important to emphasize that we obtain good performance without image pre-processing. Simple gray scale data, as in the figures, is sufficient for good performance. This is in marked contrast to other stereo algorithms, which require edge enhancement or feature detection in order to perform at all.

Small regions of the image which have no spatial detail will off course fail to yield a disparity signal. This is true of any matching algorithm.

\section{Robustness of algorithm}

We have found the columnar cepstral algorithm to resist a wide range of image degradations. Figure 5A shows a pair of image patches (random dot stereogram) in which a Gaussian blur was applied to the right frame, and then some random "scribbling" was added. The thresholded cepstrum is shown below. Figure 5B shows a pair of natural images, with the same Gaussian blur and scribbling applied. The thresholded cepstrum is also shown beneath it. Figure $5 \mathrm{C}$ is a natural image pair, in which

\footnotetext{
${ }^{2}$ Humans do not see aliased edges in random dot stereograms (based on our subjective experience), but merely cannol resolve fine positional detail, below aboul 10 minules of arc, in pure slereo images. Stereo fusion, which we have not addressed, would need to be considered to complete the segmentation algorithm presented in this paper, and it would be of interest to compare the subjective qualities of such a fusional algorithm with human performance.
} 


\section{CEPSTRAL FILTERING}

the left frame was histogram equalized, and the right frame was not, with the thresholded cepstrum shown below. Size changes of up to $15 \%$ and rotations of ten degrees of one of the stereo frames can be routinely accepted by this algorithm. Considerable intensity changes can be applied to one of the stereo frames without disrupting the algorithm. In figure 5, we show an example in which one of the stereo pairs is "histogram equalized" and the other is left in its original ( low contrast) state. The algorithm of the present paper was not disturbed by this intensity difference, nor by simple additive intensity increments of $50 \%$ to one image of a stereo pair. In fact, positive and negative stereo pairs can be processed with no difficulty, as is evident from the mathematical structure of the cepstral filter. ${ }^{3}$

\section{Relation to other correlational methods}

The cepstral filter is closely related to auto-correlation. The question naturally arises whether auto-correlation would perform as well as the cepstral filter in this application. Similarly, does simple cross-correlation of two stereo scenes perform well?

Our experience with auto-correlation applied to a columnar architecture, using the same data with cepstral filtering, is that the cepstral filter is superior. The reason for this is that there can be interference between spatial frequency terms in the half-images and the disparity term in conventional correlational approaches. One could avoid this only by passing the stereo frames through a low pass filter, which would remove all image power at high frequencies ( comparable to the range of disparities of interest).

The cepstral approach avoids this problem, since the disparity terms and the image specific terms in the cepstrum are well separated. Thus,the cepstral filter is optimized for the present application. Since its computational cost is virtually identical ( both in complexity and in reality!)to auto-correlation , and its performance on columnar images is clearly superior, it is the method of choice.

\footnotetext{
${ }^{3}$ Humans can fuse stereo pairs which differ considerably in intensity, and can fuse positive-negative pairs[13]. Similarly, humans can fuse slereo pairs which have size differences of up $1015 \%$ and rotational differences of up 1015 degrees. In these respects, the cepstral algorithm has similar robustness to human stereo vision.
} 


\section{Discussion}

Recent approaches to stereo segmentation (see[11] for review) typically have a sequential, iterative, or relaxation component. Thus, some search procedure ( e.g. relaxation, or search over multiple scales ) is used to resolve ambiguities in local image matches. As Marr[2] has pointed out, these approaches do not seem biologically plausible: the ability of biological systems to function in extremely short time intervals seems to argue against elaborate variational, cooperative, or relaxation processes. In fact, any iterative step at all seems questionable when considering the performance of pre-attentive segmentation in humans: a time period of about $200 \mathrm{msec}$ is enough for a complete pre-attentive segmentation of a complex scene. Yet, $200 \mathrm{msec}$ is about the same amount of time it takes a signal to propagate through all layers of visual cortex, from the retina. The implication is that pre-attentive segmentation in humans is a "one-shot" procedure, since it seems to occupy little more than a single pass through the cortical "machine".

The cepstral algorithm described in this paper is purely parallel, so is not subject to Marr's critique of iterative algorithms. Without columnar image format, this algorithm would be problematic. If the two images were simply superimposed (e.g. by addition), the performance would be severely degraded, since there would be no clear "echo" signal. Moreover, the very small disparity terms in the cepstrum would be masked by neighboring frequency components in the images.

\section{Limitations of this algorithm}

The principal limitation of this algorithm is that, in so far as it is a "textural" approach, it can only operate in windows of size greater than the resolution limit of the image.

Ocular dominance columns are perhaps five times larger than the basic acuity limit of the visual system. This is a comfortable range for our window operator, and is in agreement with psychophysical measurements of the spatial limits of disparity sensitivity in humans.

Other algorithmic approaches, based on local feature matches for example, might be capable of extending this limit to within the range of acuity. However, they do so at considerable computational expense, and would, in the process, exceed the actual abilities of the human visual system. (We are 


\section{CEPSTRAL FILTERING}

implicitly considering the performance of human vision as optimal in this context, rather than consider an unconstrained definition of the term "vision").

\section{Biological implementation and relation to 'spatial frequency channels'}

The method of implementation which we have used to simulate the cepstral filter requires little more than access to the spatial frequency content (power spectrum) of an interlaced stereo pair. For many years, there has been considerable interest in the "spatial frequency" tuning properties of the human visual system. It is interesting to note that an appropriate set of band-pass frequency filters are adequate to implement the cepstral filter of this paper.

It is important to emphasize that access to a fourier transform is not necessary for the present algorithm. Thus, phase information is not required. Estimates of power spectral density, which could be provided by simple space domain filtering are sufficient.

We have not yet studied the implications of cepstral filtering in a biological context. Nevertheless, it is interesting to point out that a recursive application of spatial filtering, as in the cepstral filter, can have powerful image processing capabilities if the underlying architecture ( e.g. columns) is correct. Finally, a prediction associated with the present algorithm is worth mentioning. Although binocularly tuned neurons are known to exist in primate visual cortex[14], it is not known whether there is any systematic spatial organization (e.g. disparity columns). An arrangement of disparity tuning in a direction perpendicular to the boundaries of ocular dominance columns would be a strong support for the cepstral filtering mechanism proposed in this paper.

\section{Generalization to other columnar systems}

The columnar architecture which is at the basis of the present algorithm is common in the primate neo-cortex. Orientation of edges ${ }^{4}$ and direction of motion, are two other modalities which are known to be formated in columnar terms. Color and spatial frequency have been claimed to have columnar architecture, and regions of frontal cortex with unknown function are also known to have a

\footnotetext{
4Te orientation column system of primate conex would provide, when operaled upon by the cepstral algorithm, a measure of boundary curvature:difference of orienlation is curvalure. Thus, slereo and boundary curvature exlraction could be provided by the same underlying mechanism, operating on the respective columnar syslems of ocular dominance and orientation selectivity.
} 


\section{Yeshurun Schwartz}

columnar architecture [15]. In earlier work, the frequency modulation aspect of ocular dominance columns was pointed out[16], and it was suggested that columnar systems occur in cortex whenever two slightly different modalities need to be compared, and their differences extracted. The cepstral operator is sufficiently simple, and the columnar architecture sufficiently widespread, to provide a hope that perhaps a generic operation of the visual system is provided by the analysis of this paper. If so, then support would be provided for the notion that the visual system uses the elaborate functional architecture which it has constructed for computational purposes.

\section{Space variant vision}

In order to make effective use of the algorithm of this paper, it is necessary to use windows which approximate the size of a pair of human ocular dominance columns. This is estimated to be about 3 - 6 minutes of arc ( foveally). It is reasonable to require perhaps 20 pixels in such a window. Thus, $512 \times 512$ pixel image would span only a few degrees. Clearly, a much higher resolution sensor than $512 \times 512$ is desirable if any appreciable angular extent is to be covered. ${ }^{5}$ An interesting alternative would be to use a space variant sensor ( i.e. a foveal sensor), patterned after the human visual system[12]. This would allow a wide angle of coverage, but still provide a reasonable allocation of sensor resources, mimicking the approach of biological visual systems. In other work, we have begun to study the image processing aspects of such space variant systems ( see figure 6 for a computer simulation of a natural scene, imaged by a space variant system such as primate visual cortex $)[17,18]$. It is amusing to note the possible synergy between the biologically motivated windowed cepstral filter, and the need to consider space variant (e.g. logarithmic) visual systems.

\section{Conclusion}

Computation consists of algorithms applied to data structures. By means of formating visual data, in the brain, in terms of adjacent "columns", a disparity signal is modulated onto a particular component of the cepstrum of the cortical image. Thus, good data structure (columnar interlacing) allows a

${ }^{5}$ Note that the random dot stereogram of figure 4 was of size $3500 \times 3500$, in order to cover 8 degrees of simulated field at 5 minute/window resolution! 


\section{CEPSTRAL FILTERING}

simple algorithm ( cepstral filter) to provide a robust solution to a computationally intensive application.

A possible lesson from biological vision may be the importance of using simple algorithmic procedures, applied to novel spatial architectures. At the same time, there is a trade off of positional resolution for discrimination: humans can discriminate stereo differences with extra-ordinary accuracy ( stereo-acuity), but cannot resolve high spatial frequencies in the stereo modality.

These design choices, which are not intuitively obvious, are the product of an extremely long "burn in" : evolution. Perhaps robotics vision applications can profit from this process. 


\section{Appendix A}

\section{Multiple disparities due to large windows}

The log power spectrum of an interlaced image pair is given by:

$$
\log F(u, v)=\log S(u, v)+\log \left(1+e^{-i \pi D \cdot u}\right)
$$

The power spectrum of this image is the cepstrum. We show that the second term of equation (1) above consists of a series of delta functions whose spatial location in the cepstrum is a measure of the shift $D$.

Using the expansion $\log (1+z)=\sum_{1}^{\infty}(-1)^{n+1} \frac{z^{n}}{n}$, valid for $z \bar{z} \leq 1$, and $z \neq-1$, we can evaluate the fourier transform

$$
\int_{-\infty}^{\infty} e^{i \pi X u} \log \left(1+e^{-i \pi D \cdot u}\right) d u=\sum_{1}^{\infty}(-1)^{n+1} \frac{\delta(X-n D)}{n}
$$

Thus, we get delta functions at locations which are integral multiples of the shift term $D$. The weight of these delta functions decreases rapidly, and, in particular, there is only a single delta function in the interval $\left[\frac{D}{2}, \frac{3 D}{2}\right]$.

2. Multiple disparities due to the columnar architecture

The situation is best illustrated graphically. Consider the table below: 


\section{CEPSTRAL FILTERING}

\begin{tabular}{|c|c|c|c|c|c|c|c|}
\hline L0 & $\mathrm{R} 1$ & L1 & R2 & L2 & d1 & d2 & actual shift \\
\hline$\overline{x x x x x x}$ & 123456 & 123456 & $789 \mathrm{ABC}$ & $789 \mathrm{ABC}$ & 6 & - & 0 \\
\hline$x \times x \times x 1$ & 123456 & 234567 & $789 \mathrm{ABC}$ & $89 \mathrm{ABCx}$ & 5 & 1 & -1 \\
\hline$x \times x \times 12$ & 123456 & 345678 & $789 \mathrm{ABC}$ & $9 A B C x x$ & 4 & 2 & -2 \\
\hline$x \times x 123$ & 123456 & 456789 & $789 \mathrm{ABC}$ & $\overline{A B C x \times x}$ & 3 & - & -3 \\
\hline$x \times 1234$ & 123456 & $56789 \mathrm{~A}$ & $789 \mathrm{ABC}$ & $\mathrm{BCx} \times \mathrm{xx}$ & 2 & 4 & -4 \\
\hline$\times 12345$ & 123456 & $6789 \mathrm{AB}$ & $789 \mathrm{ABC}$ & Cxxxxx & 1 & 5 & -5 \\
\hline$\overline{x x x x x x}$ & 123456 & $\times 12345$ & $789 \mathrm{ABC}$ & $6789 \mathrm{AB}$ & 7 & 13 & 1 \\
\hline$x x x x \times x$ & 123456 & $x \times 1234$ & $789 \mathrm{ABC}$ & $56789 \mathrm{~A}$ & 8 & 14 & 2 \\
\hline$x x x x \times x$ & 123456 & $x \times x 123$ & $789 \mathrm{ABC}$ & 456789 & 9 & 15 & 3 \\
\hline$x \times x \times x x$ & 123456 & $x \times x \times 12$ & $789 \mathrm{ABC}$ & 345678 & 10 & 16 & 4 \\
\hline$x x x x \times x$ & 123456 & $x \times x \times x 1$ & $789 \mathrm{ABC}$ & 234567 & 11 & 17 & 5 \\
\hline$x x x x \times x$ & 123456 & $x \times x x x x$ & $789 A B C$ & 123456 & - & 18 & 6 \\
\hline
\end{tabular}

We have constructed a schematic model of "columns" 1 unit high and 6 units wide, depicted by L0 to L2. An image is simulated in this table as a sequence of the characters "123456ABC", and " $x$ " represents arbitrary data. The table summarizes the possible disparity matches which can exist as a function of the "actual shift" of the underlying images. The complication in this simulation is due to a "wrap-around" condition which is introduced by the existence of periodic columns of interlaced image, and by considering windows of more then two adjacent columns. Thus, the complexity of this situation is due to the actual architectural complexity of the primate visual cortex.

The possible disparity values which can exist are indicated in the table as $\mathrm{d} 1$ and $\mathrm{d} 2$. If the algorithm compares only adjacent columns ( e.g R1 and L1), then a single peak will be detected by the algorithm. However, its intensity is related to the degree of correspondence between these columns: for actual shift of -1 , for example, the two columns share the part of the image represented by "23456", while for an actual shift of -4 , this part is "56" only, which will yield a weaker cepstral peak. Thus, comparing two adjacent columns, disparities up to the size of the column can be detected, but the corresponding peak is attenuated as the shift grows, until it vanishes when it reaches the size of the column.

Another point which should be addressed is the possibility that the "disparity signal" will be masked by the signal of the image (see equation 1) for large negative shifts (e.g -4 and -5 in table 1).

This could be avoided by either considering disparities in the range $\left[\frac{D}{2}, \frac{3 D}{2}\right]$ (i.e actual shift of -3 to 
3 in the table), or, as mentioned in the description of the cepstral filter, by subtracting the cepstrum of the image from the columnar cepstrum before the peak detection.

Thus, we feel that the execution of this algorithm is simplest for disparities whose range allows the shifted image terms to remain within neighboring windows. This will occur for disparities in the range of a single column width, which corresponds to about 5 minutes of arc.

Panum's area[11,6], refers to a range of stereo disparity over which human's can easily "fuse" stereo frames. It is possible for humans to process stereo at larger disparities, but special conditions are required: first, fusion is effected within Panum's area, and then the stereo frames are "pulled" slowly to larger disparities.

It is thus interesting to note that our column based algorithm has similar performance characteristics to human stereo. It is limited in positional resolution, as outlined in the text, but is also limited in the range over which it can most simply process stereo images. Both of these limitations are determined by the width of ocular dominance columns. Thus, there is a basic scale factor in human stereo, of magnitude 5 - 10 minutes arc, which determines the limit in both positional resolution for stereo, and the limiting region for easy stereo processing. Our algorithm has very similar limitations. 


\section{CEPSTRAL FILTERING}

\section{FIGURE CAPTIONS}

Figure 1. This figure shows the pattern of ocular dominance columns of a macaque monkey. The cortex has been digitally sectioned in the true tangential plane[19] it has then been numerically flattened with minimal error[20], and then the gray scale values of tissue stain have been texture mapped onto the flat cortical model.

The data in this figure were obtained from a one eyed monkey whose brain was subsequently stained with a metabolic marker ( for cytochrome oxidase). Darker regions indicate higher values of metabolism, i.e. they correspond to the present, active eye. The periodic pattem of the ocular dominance column system is clearly visible.

In the context of the present paper, each of the dark stripes represents image input from the left eye, and each of the lighter stripes represents image input from the right eye. These strips are about 400 $\mu$ wide, and correspond to perhaps 5 minutes of arc in the visual field.

Figure 2A shows a pair of image patches. There is both horizontal and vertical disparity. Figure $2 \mathrm{~B}$ shows the power spectrum of $2 \mathrm{~A}$. The origin of the frequency plane has been shifted to the center of the frame. Figure $2 \mathrm{C}$ shows the cepstrum of $2 \mathrm{~A}$. The disparity terms occur as bright spots in the cepstrum. These spots are easier to see in a thresholded version, shown in figure $2 \mathrm{D}$. The box marks a region of the cepstrum, described in the text, which does not need to be searched for a disparity signal. Figure $2 \mathrm{E}$ shows the cepstrum of the left half image of $2 \mathrm{~A}$. Figure $2 \mathrm{~F}$ shows the subtraction of the left cepstrum from the interlaced cepstrum. Only the two disparity "dots" remain.

Figure 3. Figure $3 \mathrm{~A}$ is one frame of a stereo pair of a natural scene. Figure 3B shows a patch of this stereo-pair, corresponding to five minutes of arc. The area of the patch is indicated by the arrow in the figure. Figure $3 \mathrm{C}$ shows the cepstrum of the interlaced patches of figure $3 \mathrm{~B}$.

Figure 4. Figure 4A shows a random dot stereogram, in which a "pac-man" figure exists as a pure stereo signal. Figure $4 B$ shows the segmented figure, after windowed cepstral filtering and peak detection. The gray scale of the segmented figure is proportional to stereo disparity. The original stereo frames were constructed at $3500 \times 3500$ pixels, and correspond, for typical reading distances, to about eight degrees of field. In other words, if the size of the box is $4 \mathrm{~cm}$, and is held at $30 \mathrm{~cm}$ from the eys, 
it will subtend 8 degrees. The window size used was 5 minutes of arc ( 32 pixels).

Figure 5. Figure 5A shows a random dot stereogram. The right image has been blurred ( Gaussian convolution), and it has been "scribbled". Figure 5B shows a small segment of a natural scene, in which blur and "scribbling" have also been applied. Figure 5C shows a small segment of a natural scene, in which the left frame has been histogram equalized, changing its intensity values. The disparity peaks are clearly evident, outside of the region between $\left(\frac{D}{2}, \frac{3 D}{2}\right)$, in each thresholded cepstrum.

Figure 6. Figure $6 \mathrm{~A}$ shows a wide angle fish eye view of a scene in the hall of our laboratory. A ladder is to the right, an eye chart is in the very center of the frame ( almost invisible). The original version of this scene was digitized to an effective resolution of $16000 \times 16000$ pixels by a polar coordinate mosaic technique. A "blow-up" of the central region of this original frame is shown in figure 6B. This is an eye-chart, and the distance to the chart was twenty-feet. In the original, line 7 of the chart could be easily read, indicated an effective "acuity" of 20/30, or about 1.5 minutes of arc.

The purpose of this work was to simulate a wide angle scene (about 100 degrees), roughly comparable to human vision, at human visual acuity.

Figure $6 \mathrm{C}$ shows this scene, blurred by a space variant filter which is modeled after human visual acuity. Figure $6 \mathrm{D}$ shows the image of $6 \mathrm{~A}$, modeled in terms of a complex logarithmic model[12] of human visual cortex. The eye-chart occupies almost half of the surface of visual cortex, although it occupies a tiny fraction of the original scene. The ladder, and the windows of the original are compressed to almost the same size as the centrally fixated letters of the eye-chart. This illustrates the tremendous space variant compression of human vision. Variations in linear size of about $100^{2}: 1$ ( $10^{4}$ in solid angle) occur from the center to the periphery of the human visual system. 


\section{CEPSTRAL FILTERING}

\section{References}

[1] D. Marr and T. Poggio, "A theory of human stereopsis," Proc. R. Soc. B 204, p. 301 (1979).

[2] David Marr, Vision, W. H. Freeman, New York (1982).

[3] L.R. Rabiner and B. Gold, Theory and application of digital signal processing, Prentice Hall (1975).

[4] S. LeVay, D.H. Hubel, and T.N. Wiesel, "The pattern of ocular-dominance columns in macaque visual cortex revealed by a reduced silver stain," J.Comp.Neurol. 159, pp. 559-576 (1975).

[5] B.P. Bogert, W.J.R. Healy, and J.W. Tukey, "The quefrency analysis of time series for echoes:cepstrum, pseudo-autocovariance, cross-cepstrum and saphe cracking.," Proc. Symp. Time Series Analysis, pp. 20S-243 John Wiley and Sons, (1963).

[6] Christopher W. Tyler, "Sensory processing of binocular disparity," pp. 199-295 in Vergence eye movements:basic and clinical aspects, ed. Clifton Schor and Kenneth Ciuffreda, Butterworths, Boston (1983).

[7] C. W. Tyler, "Spatial organization of binocular disparity sensitivity," Vis. Research 15, pp. 583-590 (1975).

[8] J. Horton, Proc. Roy. Soc. 25, (1985).

[9] B.M. Dow, A.Z. Snyder, R.G. Vautin, and R. Bauer, "Magnification factor and receptive field size in foveal striate cortex of monkey," Exp. Brain Res. 44, pp. 213-228 (1981).

[10] D.C. VanEssen, W.T. Newsome, and J.H.R. Maunsell, "The visual representation in striate cortex of the macaque monkey: Assymetries, anisotropies, and individual variability.," Vision Research 24, pp. 429-448 (1984).

[11] Bela Julesz, "Stereoscopic vision," Vis. Research, pp. 1601-1612 (1986).

[12] E.L. Schwartz, "Computational anatomy and functional architecture of striate cortex: a spatialmapping approach to perceptual coding," Vision Research 20, pp. 645-670 (1980).

[13] B. Julesz, Foundations of Cyclopean Perception, University of Chicago Press, Chicago, IL (1971). 
[14] G. Poggio and B. Fischer, "Binocular interaction and depth sensitivity in striate cortex and prestriate cortex of behaving rhesus monkey," JNeurophys. 40, pp. 1392-1405 (1977).

[15] P. Goldman and W. Nauta, "Columnar distributions of cortico-cortical fibers in frontal, associational, limbic and motor cortex of developing rhesus monkey," Brain Research 122, pp. 393413 (1977).

[16] E.L. Schwartz, "Columnar architecture and computational anatomy in primate visual cortex: segmentation and feature extraction via spatial-frequency-coded difference mapping," Biological Cybernetics 42, pp. 157-168 (difference mapping).

[17] Y. Yeshurun and E.L. Schwartz, "Space-variant image-processing IV: Contour-based blending of multi-fixation log views of a scene," Comp.Neuro.Tech.Rep. CNS-TR-11-86, NYU Med. Ctr./Computational Neuroscience Laboratories, (1986).

[18] E. Wolfson, Y. Yeshurun, and E.L. Schwartz, "Space-variant image-processing II: Imageblending of multi-fixation logarithmic views," CompNeuro.Tech.Rep. CNS-TR-10-86, NYU Med. Ctr./Computational Neuroscience Laboratories, (1986).

[19] W. Light and E.L. Schwartz, "A digital tangential microtome built from a voxel-based surface tracker: The Brain Peeler," CompNeuro.TechRep. CNS-TR-5-86, NYU Med. Ctr./Computational Neuroscience Laboratories, (1986).

[20] E.L. Schwartz and B. Merker, "Computer-aided neuro-anatomy: Differential geometry of cortical surfaces and an optimal flattening algorithm," IEEE Computer Graphics and Applications 6, pp. 36-44 IEEE Computer Society, (1986). 


\section{CEPSTRAL FILTERING}

Acknowledgement

We thank Robert Hummel and David Lowe for reading this work, and for making suggestions towards its improvement, and Nina Brennan for editorial assistance.

Page 22 


\section{FIGURE}

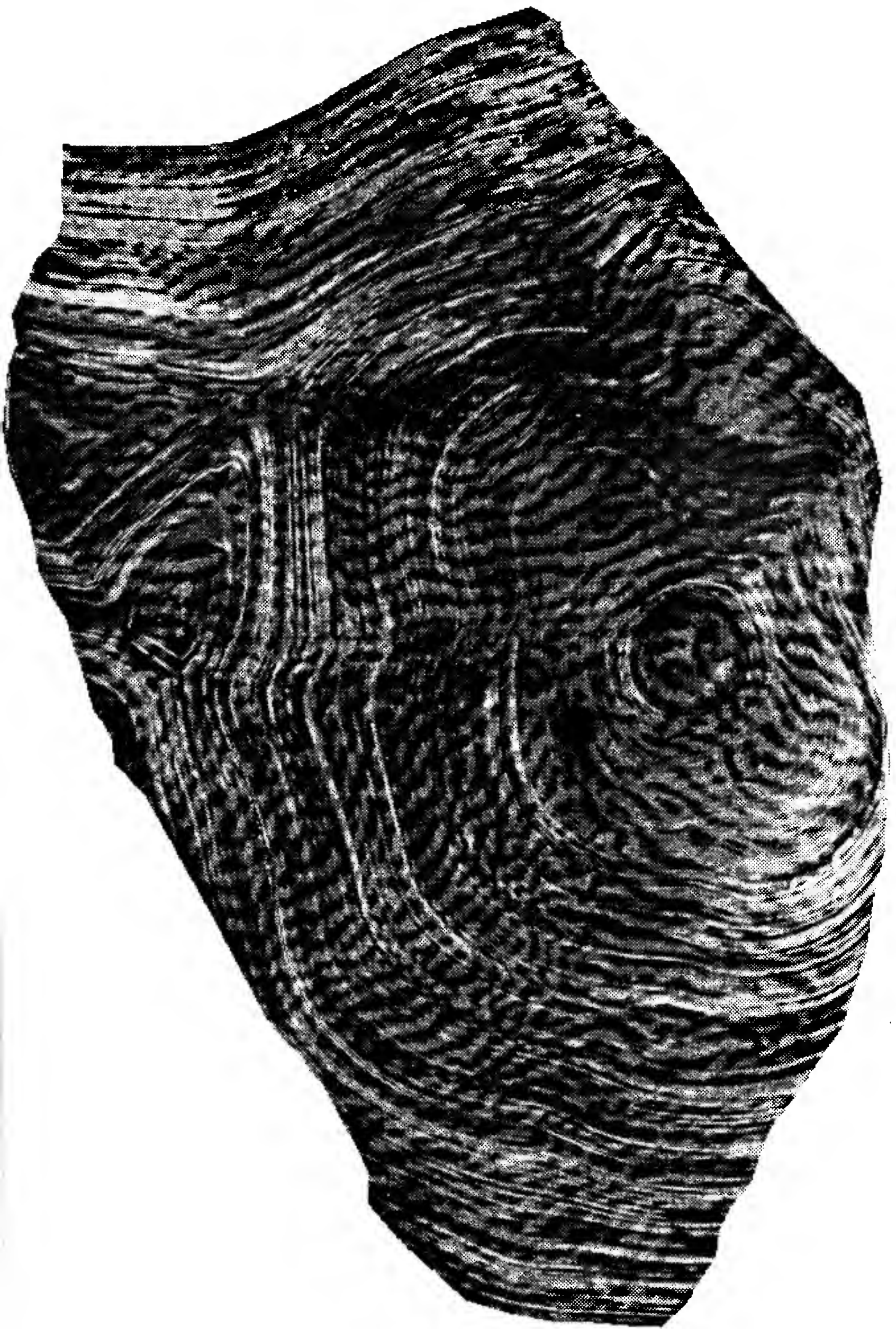


Figure 2

A

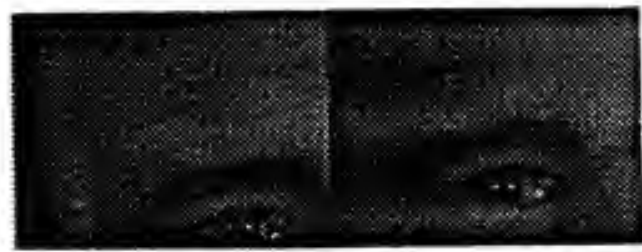

3

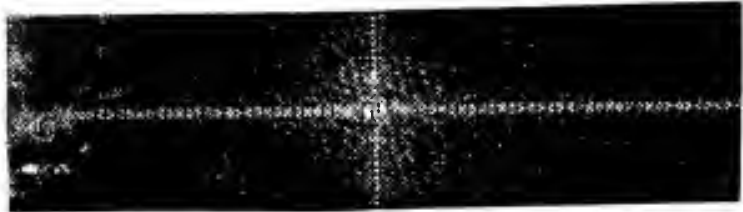

c

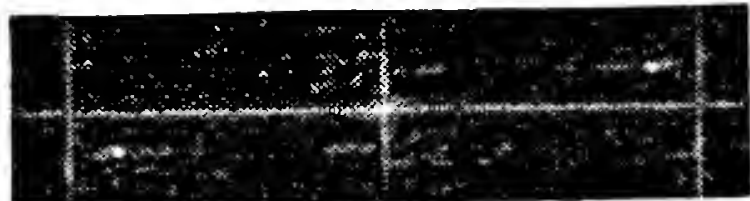

D

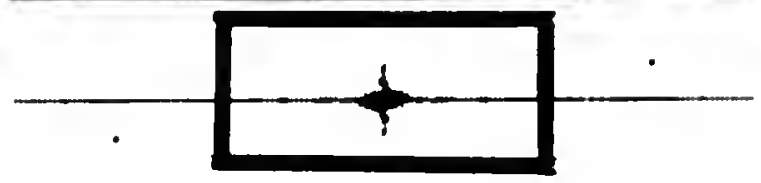

E

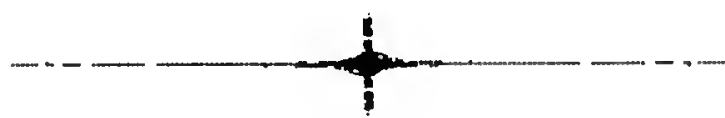

F 
Figure 3

A

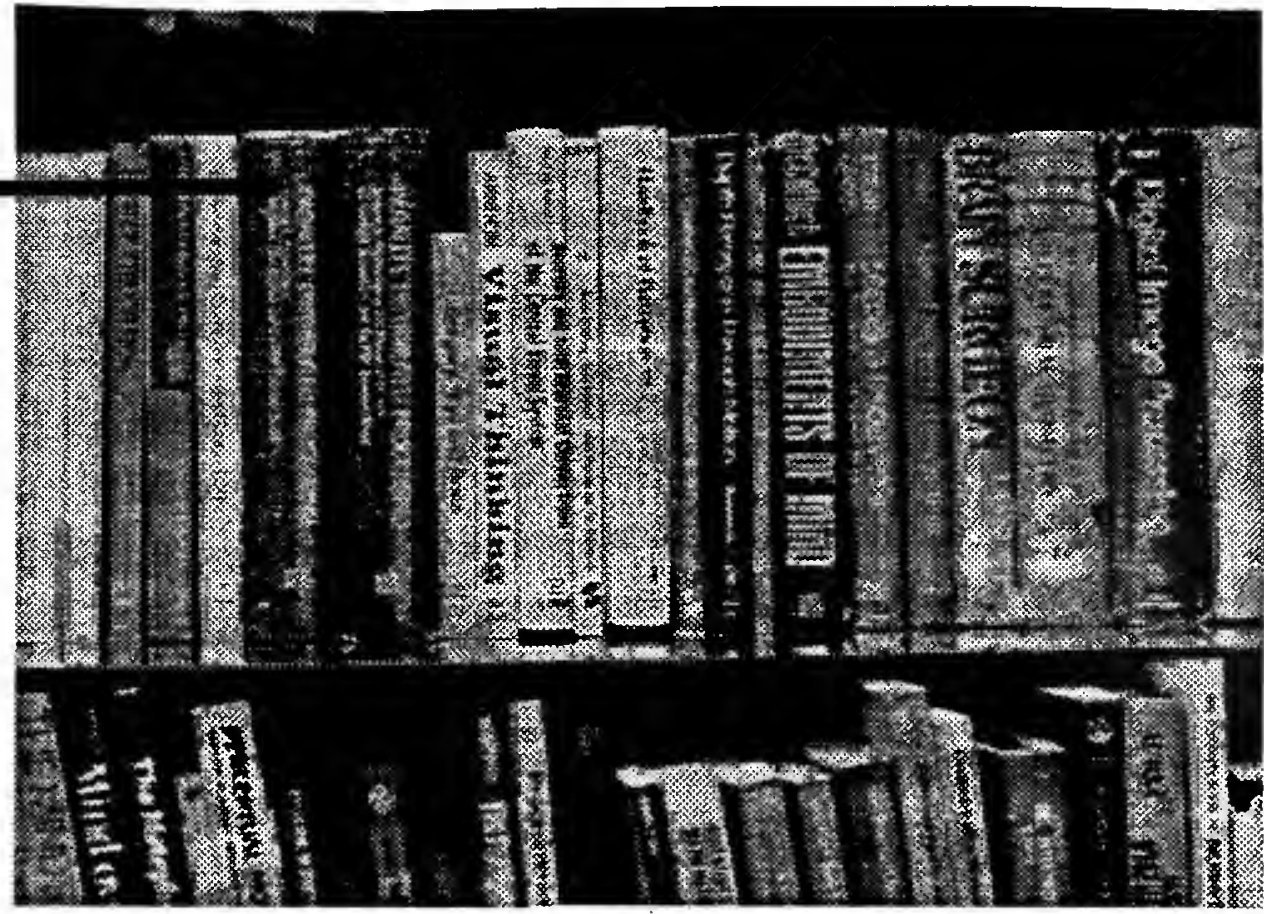

B
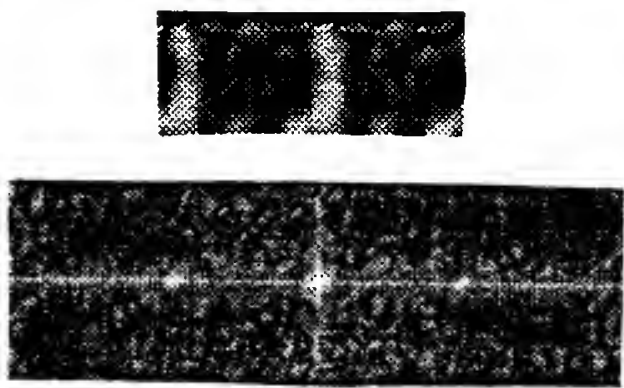


\section{Figure 4}
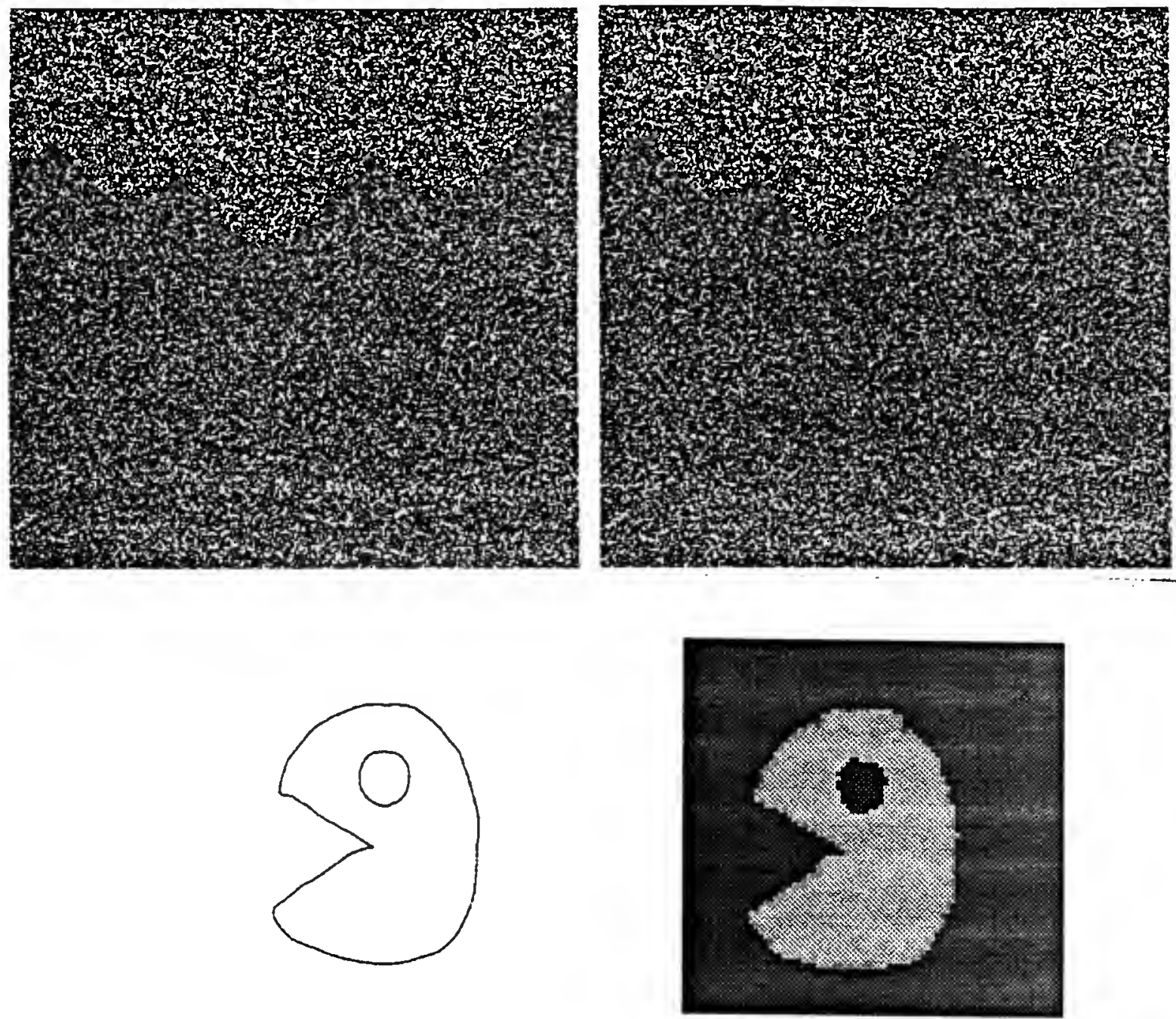
Figure 5

A

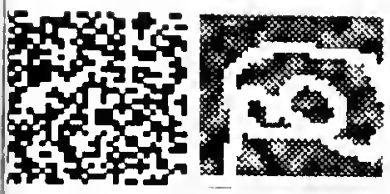

$\because$ ind $\because$.

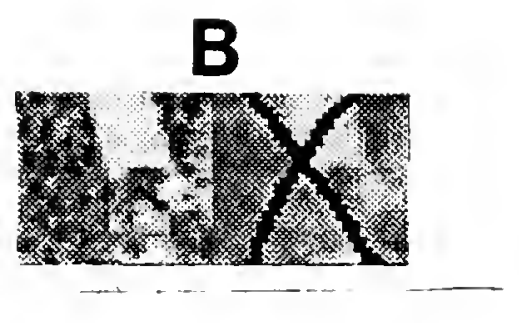

…지….

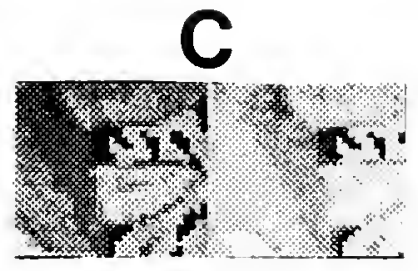

... $1 .$. 


\section{Figure 6}

A

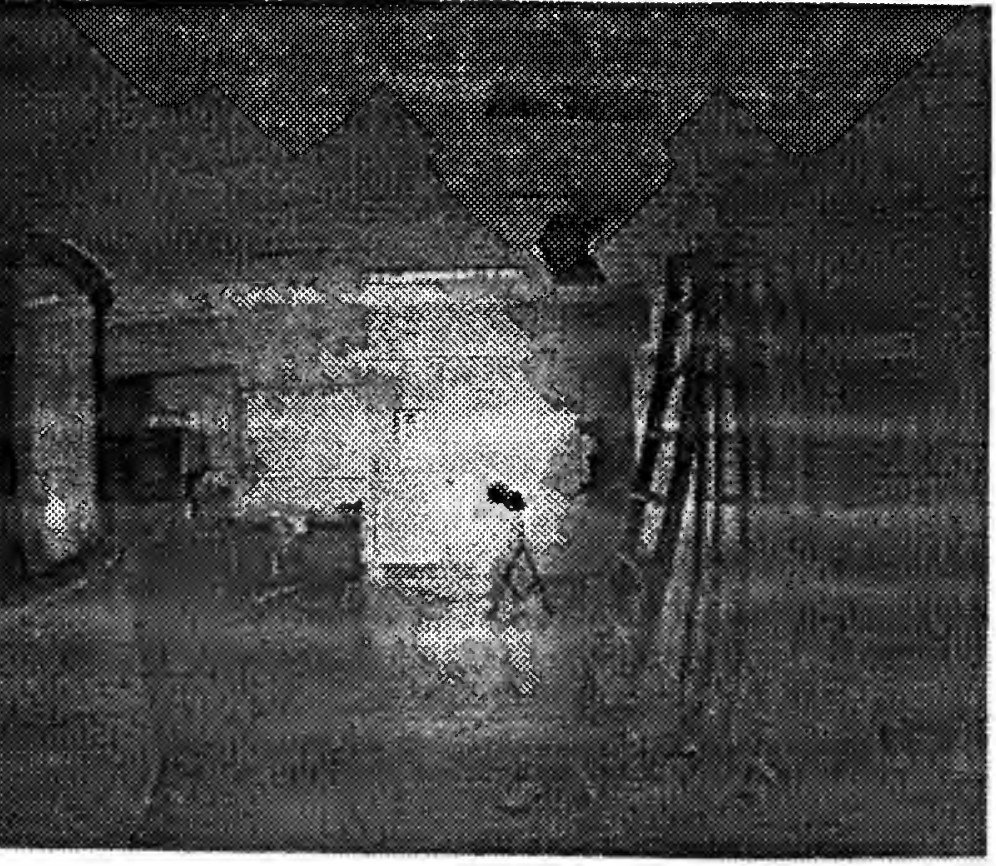

C

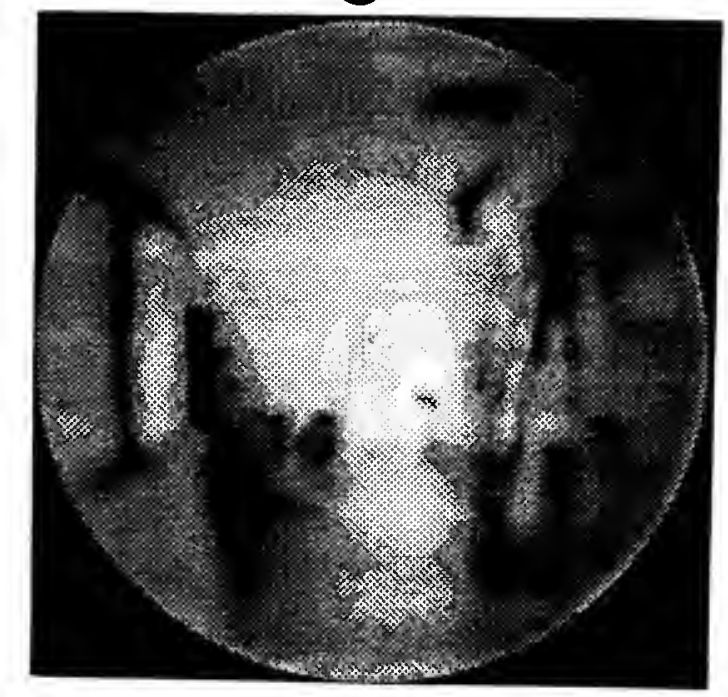

B

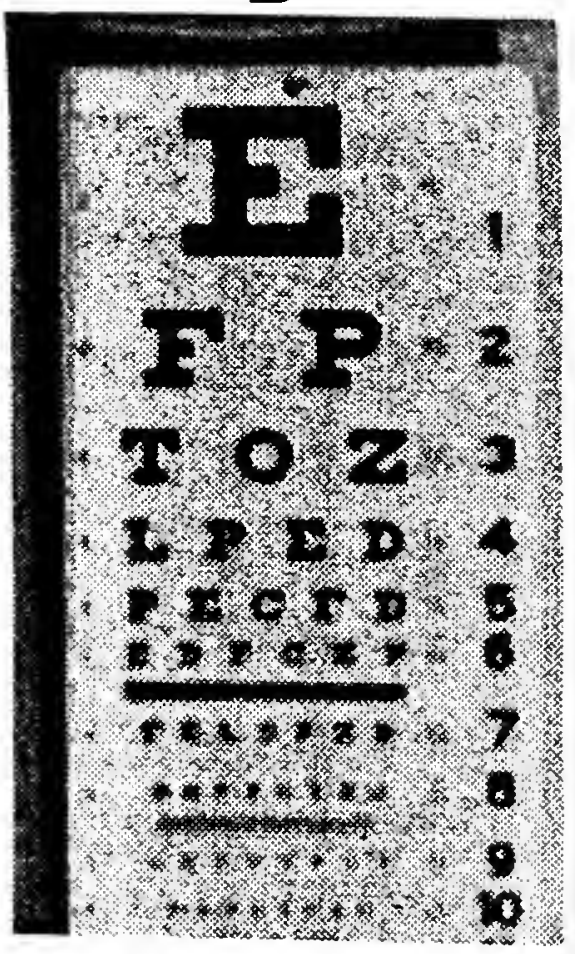

D

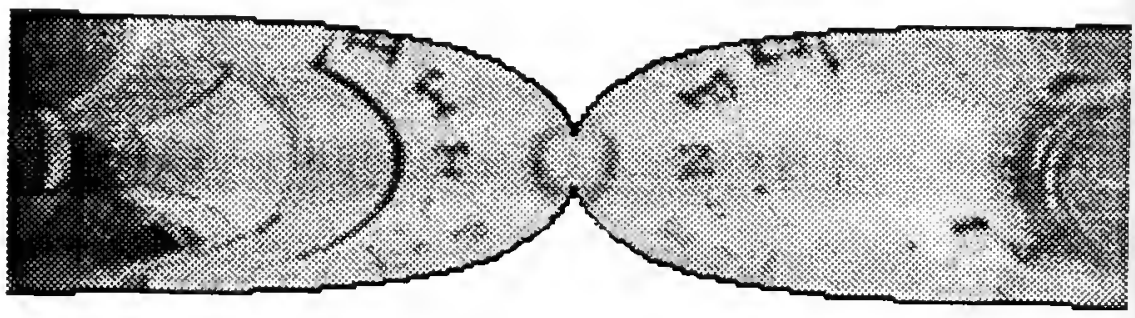


NYU COMPSCI TR-286

C. 1

Yeshurun, Yehezkel

Cepstral filtering on a columnar image

architecture

\section{LIBRARY}

N.Y.U. Courant Institute of Mathematical Sciences 251 Mercer St.

New York, N. Y. 10012 


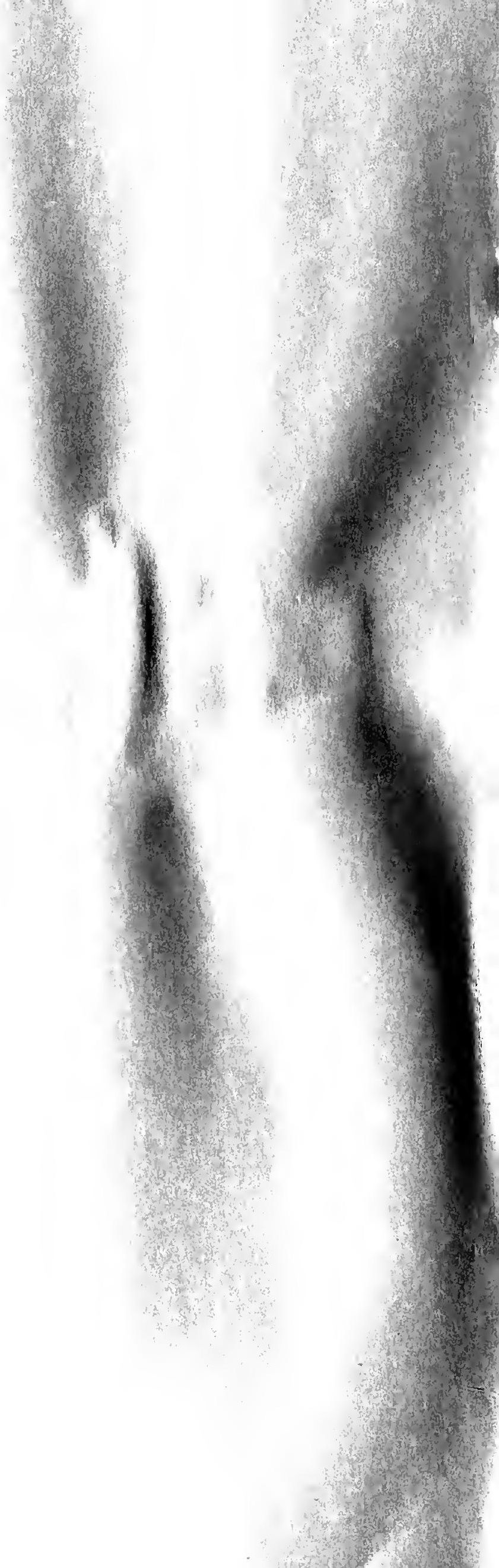

